\title{
DINÁMICAS DE LA INTERPRETACIÓN POÉTICA: EMOCIÓN Y ESTÉTICA COGNITIVA EN LA LECTURA LITERARIA ${ }^{1}$
}

\author{
DINAMICS OF POETIC INTERPRETATION: \\ EMOTION AND COGNITIVE AESTHETICS \\ IN LITERARY READING
}

\author{
Víctor BERMÚDEZ \\ Christian-Albrechts-Universität zu Kiel \\ victor.bermudez@romanistik.uni-kiel.de
}

Resumen: Este estudio explora el pensamiento poético incidiendo en la especificidad del contenido emocional de textos literarios de expresión francesa. Tras una valorización de la subjetividad lectora en el proceso de interpretación, se destaca el papel de las emociones en la generación de significado y se brinda una aproximación a la imaginación literaria. Asimismo, se elabora un análisis de vocación interdisciplinar de la serie de poemas "Nuits", de Lorand Gaspar, que aúna perspectivas neurobiológicas y filosóficas con el doble propósito de incorporar herramientas de interpretación poética a la teoría literaria y de reconocer estructuras de razonamiento de la creación literaria.

\footnotetext{
${ }^{1}$ El presente artículo se ha realizado en el seno del Proyecto de Investigación Inscripciones literarias de la ciencia: cognición, epistemología y epistemocrítica (ILICIA), subvencionado por el MINECO. Ref. FFI2017-83932-P.
} 
Palabras clave: Emociones. Poética cognitiva. Lorand Gaspar.

Abstract: This study explores poetic thought, focusing on the specificity of the emotional content of poetic texts in French language. After a valorisation of the reader's subjectivity in the interpretation process, the role of the emotions in the generation of meaning is highlighted and an approximation to the literary imagination is offered. Also, the study elaborates an interdisciplinary analysis of the series of poems "Nuits", by Lorand Gaspar, which combines neurobiological and philosophical perspectives with the double purpose of nourishing the tools of poetic interpretation of Literary Theory and of recognizing structures of reasoning of the literary creation.

Key Words: Emotions. Cognitive poetics. Lorand Gaspar.

\section{INTRODUCCIÓN}

La lectura no es un acto biológico natural. Un complejo aprendizaje media este acto, habitualmente en las etapas tempranas de la educación, modelando actitudes de acercamiento al texto cuya repercusión resulta incalculable para la interpretación de la literatura. Aprender a leer es un fenómeno excepcional de la vida que tiene hondas implicaciones para la recepción y generación de los discursos literarios. En lo que respecta a la teoría de la literatura, la atención al proceso de lectura fue un objeto de estudio en auge durante la década de 1930, como reacción a la tendencia existente a infravalorar el papel del lector en la generación del significado de la literatura. Así, desde el New Criticism se elaborará una distinción entre la forma de un texto y su efecto, reivindicando la importancia de cada acontecimiento de lectura y relativizando la intención discursiva de su autor. Acaso esta necesaria valorización de la subjetividad lectora ha 
desplazado la atención del universo textual en sí y de las posibilidades que este brinda para aproximarse a la imaginación literaria.

En el contexto del New Criticism resultan de interés cuestiones tales como las inferencias emocionales que intervienen en la comprensión de la literatura, el papel del conocimiento previo, las intenciones y motivaciones que rodean la decodificación de los textos poéticos, así como también los mecanismos de la memoria que entran en funcionamiento orientando actitudes de lectura ante la indeterminación del significado que en ocasiones plantea el lenguaje literario. De tal modo que la teoría de la literatura estudia, desde distintas perspectivas, las dinámicas de armonización y disonancia a las que somete la lectura de un texto. A partir de dichas dinámicas se analiza el procesamiento de tensiones, patrones, sintesis y constancias, así como los de flexibilidad, adaptabilidad, apertura al cambio y a la impredecibilidad; nociones que exploran la predicción y anticipación y la ruptura de la expectativa en el proceso de lectura y que describen parte del proceso de interpretación y comprensión de lo leído.

Sin embargo, la teoría literaria no encuentra pleno consenso acerca de las reacciones en la lectura y hasta qué punto estas vienen determinadas por el texto, como indica Tyson: "Opinions range from the belief that the literary text is as active as the reader in creating meaning to the belief that the text doesn't exist at all except as it is created by readers" (Tyson, 2006: 172). En este sentido, resultan diversas las perspectivas teórico-literarias que observan la interacción entre el contenido del texto y la experiencia de lectura. De tal modo, el New Criticism señalaba que lo que el texto es no puede ser separado de lo que el texto provoca (Fish, 1970: 123). Asimismo, con el término affective stylistics, el teórico Standley Fish sostiene que el significado del texto es un acontecimiento unido a la experiencia de lectura, por lo que un poema sin lector carece de significado.

Por su parte, la Reader Response Theory incide en que el lector no "consume" pasivamente un significado que el texto presenta "objetivamente" ante él, sino que aquel se encuentra activamente constituyéndolo. Así, desde una perspectiva de fenomenología literaria, Wolfgang Iser 
confiere al lector la facultad de proyectar significado sobre el texto; no obstante, Iser reconoce que las actividades lectoras a través de las cuales se construye dicho significado se encuentran "preestructuradas" por el texto mismo. Iser considera que el texto orienta los procesos involucrados en la interpretación que hace el lector, aunque no los determina completamente. Con todo, al poner el acento en el vínculo entre el sentido de un texto y el suceso de lectura, el New Criticism relativiza el valor de la forma verbal como simbolización del sentimiento humano en favor de una circunstancia de lectura específica, es decir, objeta que la hermenéutica de lenguaje opera proyectando un contenido emocional objetivable.

Vinculada a los trabajos de Louise Rosenblatt, la Transactional reader response theory sostiene que lo que se da es una serie de "transacciones" entre el lector y el texto. Rosenblatt considera que en el proceso de generación de sentido se hallan implicados tanto el texto como del lector, y hace una distinción entre los términos "texto", "lector" y "poema", en la que el primero se refiere a las palabras impresas en la página, mientras que el segundo y tercero remiten a la obra literaria que surge de la conjunción entre el lector y el texto (Tyson, 2006: 173). Así, la concepción de Rosenblatt no discrepa enteramente de la hermenéutica pues confiere relevancia semejante al acto y al objeto de lectura, pero ¿cómo se produce dicha transacción?:

As we read a text, it acts as a stimulus to which we respond in our own personal way. Feelings, associations, and memories occur as we read, and these responses influence the way in which we make sense of the text as we move through it. Literature we've encountered prior to this reading, the sum total of our accumulated knowledge, and even our current physical condition and mood will influence us as well. At various points while we read, however, the text acts as a blueprint that we can use to correct our interpretation when we realize it has travelled too far afield of what is written on the page. This process of correcting 
our interpretation as we move through the text usually results in our going back to reread earlier sections in light of some new development in the text. Thus, the text guides our self-corrective process as we read and will continue to do so after the reading is finished if we go back and reread portions, or the entire text, in order to develop or complete our interpretation. Thus the creation of the poem, the literary work, is a product of the transaction between text and reader, both of which are equally important to the process (Tyson, 2006: 173).

Con todo, Rosenblatt distingue una "lectura eferente", con un carácter analítico, de una "lectura estética" en la cual interviene un vínculo subjetivo en el proceso de lectura. La lectura eferente se centra en la información que contiene el texto como si se tratase de un almacén de datos e ideas, mientras que en la lectura estética, según es concebida por Rosenblatt: "we experience a personal relationship to the text that focuses our attention on the emotional subtleties of its language and encourages us to make judgments. [...] Without the aesthetic approach, there could be no transaction between text and reader to analyze" (Tyson, 2006: 173).

Por otra parte, entre las recientes formulaciones teóricas que se interesan por los procesos de interpretación literaria cabría destacar los estudios cognitivo-literarios que investigan, por ejemplo, las respuestas afectivas al ritmo y a la versificación en poesía. En el proceso de decodificación de un texto resulta de interés crucial el concepto de "integración conceptual" (conceptual blending) de la Blending theory (Gilles Fauconnier y Mark Turner, 2008) por cuanto describe los espacios mentales que interactúan en la generación de nuevos sentidos, cuya relevancia ha sido significativa en los campos de la lingüística cognitiva y la filosofía del lenguaje. Otros trabajos han explorado, igualmente, la especificidad de las inferencias emocionales en la comprensión de textos, entre los que han de señalarse los de Christelle Gillioz (2013, 2017), quien ha investigado " $[. .$.$] the conditions under which readers may go further than$ 
the component level and reach complex emotion representations" así como "the influence of individual differences and the nature of the information presented to readers on emotion inferences in text comprehension, in particular on the specificity of these emotion inferences" (Gillioz, 2013: v). Gillioz pone igualmente el acento en la intersección lector-texto:

Text comprehension follows from the constant interaction between the text and the readers'general knowledge in order to draw relevant inferences as to maintain coherence. All models of text comprehension agree on the role played by the information transmitted in the text as a source of text processing. Information in the text activates associated information in the readers knowledge stored in long-term memory. As the text is processed, the activation of different concepts in the text and consequently in the reader's memory fluctuates (Graesser et al., 1994, en Gillioz, 2013: 17).

Pensar en términos de modelos mentales que orientan la exégesis literaria nos sitúa en el territorio de la hermenéutica. En su obra Literary Reading, Cognition and Emotion (2012), Michael Burke dedica un capítulo al "círculo hermenéutico" de interpretación. El principio hermenéutico de que la interpretación es circular sostiene que "one can understand a text [...] only by grasping in advance the relation of a specific part to the whole in which it belongs, even if one can only arrive at a sense of the whole by working through its parts" (Armstrong, 2013: 54). Por lo que la lectura implica un procedimiento de predicción a partir de especificidades, pero se trata de una anticipación sujeta a sistemáticas rupturas de la lógica y la expectativa. Para Iser la lectura no es una sencilla adicción de signos lineales que escaneamos secuencialmente; al contrario, durante la lectura literaria, el lector no queda exento de enfrentarse a la sorpresa, a la ambigüedad o el conflicto interpretativo.

En sentido amplio, el círculo hermenéutico constituye un 
razonamiento deductivo que opera mediante un reconocimiento de patrones en la atribución de significados: el género de un texto, su estilo, sus recursos o figuras literarias, su aspecto, su extensión, su organización gramatical y léxica, etc.; elementos, todos, con los que el lector anticipa la comprensión del texto y le proporciona un sentido a este. Sin embargo, la noción de circularidad desdibuja el papel de la sorpresa y la ruptura de expectativas en el proceso de interpretación del texto literario. De ahí que, en su obra How Literature Plays with the Brain. The Neuroscience of Reading and Art, (2013) Paul Armstrong señale que:

The term hermeneutic spiral would perhaps be more accurate. The to-and-from movements of interpretation can make it an unpredictable activity-an important example of the brain's proclivity to "play." Heidegger emphasizes the hermeneutic circle's temporality when he argues that interpretation is inherently futural, always guided by an anticipatory understanding that projects a range of meanings that the state of affairs in question may have, what he calls the "forestructure" (Vorstruktur) of interpretation. To interpret is therefore a process of "catch-up" (Armstrong, 2013: 56).

Con todo, es convicción de este estudio que las emociones no solamente median en la decodificación de lo leído, sino que, en no pocas ocasiones, la escritura misma encuentra arraigo último en la experiencia emocional. La importancia de la lectura y su subjetividad implícita no ha de desplazar la consideración de que el texto pueda ser comprendido a partir de su tratamiento, explícito o no, del contenido afectivo. Por ello, resulta plausible proponer indagaciones hacia el pensamiento poético que ponderen, desde la exégesis del texto, estructuras de razonamiento de la creación literaria. 


\section{ESTÉTICA COGNITIVA EN “NUITS” DE LORAND GASPAR}

El filósofo estadounidense Mark Johnson ha elaborado una indagación acerca de las distintas dimensiones del significado que se generan a partir de la relación que el cuerpo humano establece con el mundo. En su obra The Meaning of the Body: Aesthetics of Human Understanding (2007), heredera de la pragmática estadounidense del siglo XIX, Johnson adopta la perspectiva de una filosofía del lenguaje que se sirve de herramientas fenomenológicas, neurobiológicas, psicológicas y lingüísticas: dicha indagación fundamenta lo que podría denominarse una "estética cognitiva", es decir, una estética de la cognición o incluso de la conciencia. Su interés es examinar los procedimientos de la generación de significado a partir de la interacción que el ser humano tiene con el medio que lo rodea, y las consecuentes implicaciones que ello conlleva en su desarrollo psicológico, lingüístico, emocional y estético. Johnson sostiene que el significado posee un arraigo que está más allá de lo lingüístico y que es "más profundo" que los conceptos mismos.

A lo largo del pensamiento filosófico, la dicotomía histórica entre lo emocional y lo cognitivo - entendido en términos de silogismos y secuencias proposicionales - ha dificultado estimar la importancia que tienen las emociones en la generación de sentido. En el contexto de esta dicotomía Johnson adopta una posición filosófica sugerente sosteniendo que "there is no cognition without emotion" (2007: 9), y desde esta idea Johnson construye su "teoría del significado encarnado" - The Embodied Theory of Meaning-, orientada como contraargumento de que el significado se fundamente en un entrelazamiento consciente de conceptos y proposiciones, que obedecen a secuencias lógico-silogísticas. En cambio, Johnson se interesa por los aspectos no conscientes mediante los cuales los seres humanos dan sentido a las diversas dimensiones de la existencia. El enfoque del filósofo estadounidense es de una explícita inspiración fenomenológica, pues tal como señala: 
The idea that meaning and understanding are based solely on propositional structures is problematic because it excludes (or at least hides) most of what goes into the ways we make sense of our experience. In striking contrast to this conceptual-propositional view of meaning and knowledge, a substantial body of evidence from the cognitive sciences supports the hypothesis that meaning is shaped by the nature of our bodies, especially our sensorimotor capacities and our ability to experience feelings and emotions. If we look at prelinguistic infants and at children who are learning how their world works and what things mean to them, we will find vast stretches of embodied meaning that are not conceptual and propositional in character, even though they will later make propositional thinking possible (Johnson, 2007: 9).

Mark Johnson aborda cuestiones relativas a los procesos de la categorización a partir, por ejemplo, de la fenomenología del movimiento, donde apoyado en teóricos como Maxime Sheet-Johnstone, destaca la importancia del movimiento corporal como base del significado. Asimismo, incursionará en el asunto de la categorización por vía de los conceptos y perceptos, es decir, de lo que entiende como el paso del "significado encarnado al pensamiento abstracto"; sirviéndose para ello de la lingüística cognitiva — George Lakoff, Rafael Núñez- y de la neurociencia - Vitorio Gallese, Gerald Edelman, etc.- - Al hilo de este entrecruzamiento transdisciplinar, se pone de relieve que las emociones son concebidas como un complejo ensamblaje de estructuras cognitivas, procesos cerebrales y respuestas corporales.

Lo que Johnson subraya es que solo una mirada holística a lo que es la experiencia emocional desde un punto de vista químico, neuronal, fisiológico, filosófico, lingüístico, etc., puede dar cuenta adecuada de su implicación en la generación de significado. Ello sin perder de vista también su dimensión social y cultural, a la cual ha atendido igualmente 
la investigación desde diferentes paradigmas epistemológicos. Y es que una gran variedad de disciplinas se ha ocupado de abordar el papel de las emociones en el desarrollo humano. Así se observa en las aproximaciones de psicólogos como Richard Lazarus, Paul Ekman, Nico Frijda y Robert Zajonc, filósofos como Robert Salomon, Martha Nussbaum y Richard Wollheim, e incluso antropólogos como Rom Harré y Catherine Lutz. Un aspecto que tienen en común estos estudios es su interés tanto por la dimensión "individual" como por la "social-colectiva" de la experiencia emocional. El hecho de que el suceso emocional sea visto como un complejo entramado de diversas dimensiones de la existencia posibilita la investigación sobre el significado que el sentimiento y la emoción propician en el sujeto.

Existe un principio de dinamismo que subyace a la posibilidad del pensamiento. Johnson sostiene que el significado deriva de la interacción del ser humano con el medio que lo rodea. Tal como señala, "the meaning of a specific aspect or dimension of some ongoing experience is that aspect's connections to other parts of past, present, or future (possible) experiences. Meaning is relational. It is about how one thing relates to or connects with other things" (2007: 10). Esta idea de que el significado es relacional y está "conectado" a nuestra experiencia de las cosas resulta interesante en nuestra incursión en la poesía de Lorand Gaspar, donde se observa que la articulación de los elementos que interactúan en su escritura posee, en efecto, un sentido gracias a su "correlación" con el sujeto que los concibe. La realidad se elabora en el espacio poemático gaspariano como una auténtica concatenación de "objetos significantes" en la cual como sostiene Johnson - "the meaning of a thing is its consequences for experience" (2007: 10).

La teoría de Johnson no excluye categóricamente el significado conceptual y proposicionalmente codificado, pero sí señala que este es "merely the more conscious, selective dimension of a vast, continuous process of immanent meanings that involve structures, patterns, qualities, feelings, and emotions" (2007: 10). Se trata, además, de una teoría del 
significado encarnado porque "it situates meaning within a flow of experience that cannot exist without a biological organism engaging its environment. Meaning emerge "from the bottom up" through increasingly complex levels of organic activity; they are not constructions of a disembodied mind (2007: 10). Resulta sugerente la idea de que el significado emerja como consecuencia de una relación espacial "desde abajo", es decir, desde la especificidad del vínculo que el sujeto establece con la complejidad del mundo. De tal modo, el significado no puede por menos que encarnar la complejidad misma de esas interacciones del sujeto con el medio.

Cabe entonces considerar qué tipo de articulación propician la emoción y el sentimiento en tanto que vehículos del pensamiento. La mirada de Johnson al respecto es concluyente: "before there is abstract thinking, before there is reasoning, before there is speech, there is emotion" (2007: 53). El filósofo no solo insiste en diluir la dicotomía establecida entre la emoción y la cognición — proposicional_, sino que procura explicar una mediante la otra; de hecho, sostiene que "emotion and feeling lie at the heart of our capacity to experience meaning" (2007: 54). En el fondo de su argumento subyace toda una consideración epistemológica acerca del papel de las emociones en el conocimiento; dicha cuestión, crucial para la teoría de la cognición encarnada, encuentra fundamento en la base neurobiológica de la experiencia emocional.

Las investigaciones de Antonio Damasio y Joseph LeDoux respaldan una noción de significado que conlleva procesos corporales noconscientes "that help make possible our more conscious acts of meaningmaking, such as our use of language [...] meaning at this level is intimately tied to emotions, only some of which rise to the level where there is a conscious feeling of the emotion as having a meaning" (Johnson, 2007: 57). A título de ejemplo, la homeostasis es un mecanismo automatizado no consciente mediante el cual un organismo "monitorea" y "autorregula" los cambios en sus estados corporales internos. Para Johnson, las operaciones no conscientes de la experiencia implican una repercusión relevante 
en nuestra relación del sujeto con el medio que lo rodea, y dan lugar a procesamientos no conscientes del sentido; por su parte, las reacciones emocionales son procesos:

[...] arising from the perception of ongoing changes within an organism that require some transforming activity, either to continue the harmonious flow of experience or to help reestablish equilibrium in response to a perceived imbalance or disruption within the organism. Functionally, emotions evolved to evoke changes within the organism and motivate it to act in ways that tend to be conductive to its welfare (Johnson, 2007: 58).

Así, Johnson asume el reto de ofrecer una definición de "emoción" y de "sentimiento" que responda tanto al valor filosófico como al neurocognitivo de los términos. Y es que la definición de "emoción” varía tanto como las disciplinas que la investigan. De este modo, el primer asunto crucial consiste en distinguir "emociones" de "sentimientos". Siguiendo a Damasio, Johnson considera que las respuestas emocionales anteceden a la consciencia del sentimiento. A diferencia de los sentimientos, que son "consciously experienced bodily processes", las respuestas emocionales pueden operar "beneath the level of consciousness" (2007: 59), en un intervalo que oscila entre "powerful passions" y "faint feelings", y de las que los seres humanos somos conscientes en el mismo grado y medida en que se manifiesta su intensidad en nuestro cuerpo.

No obstante, "the whole neural, chemical, and behavioural arc of emotional response runs more or less automatically most of the time" (2007: 59), y esto es lo que caracteriza al significado, en sus primeros estadios de generación, como una operación que podríamos también decir "por debajo del nivel de la conciencia". Asimismo, Johnson incide en el carácter "cotidiano" de las emociones como operaciones que conducen nuestro desarrollo social y ambiental a partir de respuestas incesantes a estímulos cotidianos. Ciertamente, el filósofo atribuye un aspecto de 
"supervivencia biológica" a las respuestas emocionales que guían nuestra cotidianidad, ya sea como reacciones a situaciones de peligro o como consecuencia de un proceso adaptativo del organismo al medio.

Por otro lado, la experiencia emocional posee un cierto carácter evaluativo, pues "every emotional response is part of a process in which there is some appraisal of how a given emotionally competent stimulus stands in relation to the potential well-being of the organism" (2007: 60). Esta característica "conjetural" resulta relevante si se tiene en cuenta que las respuestas emocionales "are not merely bodily, after-the-fact feeling reactions. Rather, they are bodily processes (with neural and chemic components) that result from our appraisal of the meaning and significance of our situation and consequent changes in our body state [...]. It is in this sense that emotional responses can be said to move us to action" (2007: 61-62). Ya sea de manera consciente o no consciente, las reacciones emocionales actúan como elementos de "medición" de las situaciones a las que se enfrenta el sujeto. Johnson asienta la consideración de que la experiencia emocional puede constituir un auténtico fundamento de conocimiento. Subscribe, a su vez, la hipótesis de inspiración spinoziana de que un sentimiento es "an idea of the body". No obstante, Johnson se apoya tanto en la neurobiología como en la filosofía pragmática estadounidense para sostener que las circunstancias que rodean a los sujetos constituyen locus of emotions dinámicos.

Ahora bien, dado que el interés de Johnson es elaborar una serie de teorías acerca de la emoción, el cuerpo y el entorno, conviene aproximar sus consideraciones sobre el significado encarnado a los métodos de la teoría y el análisis literario. Con ese propósito se abordarán a continuación algunos extractos de la obra Patmos et autres poèmes de Lorand Gaspar, y concretamente de "Nuits". Se examinará ahí lo que podría denominarse una "prosodia de las emociones", que resulta un rasgo estético característico de la expresión poética de "Nuits". Además de considerar el término "prosodia" en su sentido literal, es decir, en tanto que "estudio de los rasgos fónicos que afectan a la métrica, especialmente de los acentos y 
de la cantidad" (DRAE), se recupera la palabra en un sentido figurado, para aludir al ritmo que imprime en la escritura su expresión de contenido emocional. Esto con el propósito de sostener que en fondo de la escritura gaspariana puede identificarse una estructura emocional. Al hilo de ello, se retomarán algunas consideraciones de Johnson en torno a la cognición encarnada, y a los mecanismos neurobiológicos en ella implicados. Atiéndase a este ejemplo:

on pense toucher le fond

mais la nuit n'a pas de fond

ni toit ni murs ni fenêtres -

(N'est-il pas vrai cependant que le peu

de clarté que l'esprit peut entrevoir

est nécessaire pour connaître l'obscur?)

Et je me rappelle ces nuits lointaines

(qu'une fois de plus embrasait la guerre)

sous la fragile clarté d'une lampe

trois femmes et un homme

cherchant à recoudre des corps

que d'autres au dehors sans relâche déchiraient -

(Gaspar, 2001: 149) 2\&3

Podríamos aproximarnos al texto distinguiendo dos momentos diferenciados. El primero de ellos ocuparía la primera y segunda estrofa y concentraría la carga perceptiva de la escena. La tercera estrofa, por

\footnotetext{
${ }^{2}$ Todas las traducciones aquí presentes son de mi autoría.

3"creemos tocar el fondo / pero la noche no tiene fondo / ni techo ni muro ni ventanas // (Sin embargo ¿no es cierto que la poca / claridad que la mente puede vislumbrar / es necesaria para conocer lo obscuro?) // Y recuerdo esas noches lejanas / (que una vez más abrasaba la guerra) / bajo la frágil claridad de una lámpara / tres mujeres y un hombre / intentando volver a coser cuerpos / que otros, afuera, deseaban sin descanso -" $(P A, 149)$.
} 
otra parte, acentuaría su relieve emocional. En este ejemplo se halla no únicamente un efecto de complementación perceptiva, sino de una auténtica simulación. Precisamente para apuntar este rasgo de "Nuits" cabe observar, junto a Johnson, que:

[...] mirror-neuron research supports the hypothesis that imagination is a form of simulation. Research by Marc Jeannerod (1994) shows that imagining certain motor actions activates some of the same parts of the brain that are involved in actually performing that action. Imagining a visual scene also activates areas of the brain that would be activated if we actually perceived that scene (Kosslyn, 1994, en Johnson, 2007: 162).

La consideración de que el pensamiento opera mediante simulación se ejemplifica en el poema que nos ocupa desde la concepción lírica de que "on pense toucher le fond / mais la nuit n'a pas de fond / ni toit ni murs ni fenêtres". El plural "on pense" delata que en la escena se encuentran al menos dos presencias líricas. Por su parte, el verbo "on pense" invoca la conjetura imaginaria que recrea la acción de "toucher" antes de que esta, en efecto, se produzca. Asimismo, el entorno paisajístico que rodea a los observadores líricos se presenta inasible y ello alimenta una atmósfera de perplejidad en la que el sujeto lírico se halla en un espacio indefinido, sin "fond, ni toit ni murs ni fenêtres". El hecho de "concebir" el contacto físico por vía de la evocación del acto de "toucher le fond" constituye un indicio de un gesto "real", de un suceso anticipado y de algún modo presente; "simulado", entonces.

Sin embargo, la simulación no solamente se sugiere ahí, sino también en esa otra acotación entre paréntesis que indica el ejercicio de complementación visual: “(N'est-il pas vrai cependant que le peu / de clarté que l'esprit peut entrevoir / est nécessaire pour connaître l'obscur ?)"; pero no se trata de un reconocimiento puramente visual de "le peu de clarté", sino de un verdadero "tacto de la noche", que ejerce un 
observador lírico que busca "connaître l'obscur" desde el contacto físico, gestual ("toucher le fond"). En ese espacio donde "l'esprit peut entrevoir" no sólo la "clarté", sino el "tacto de la noche", se produce un ejercicio de recuperación mediante el cual opera un aspecto de la memoria sensorial —"je me rappelle" dirá más adelante el sujeto lírico—. Lo perceptivo — lo que "l'esprit peut entrevoir" - es aquí parte de un mecanismo más amplio no sólo de recuperación, sino de verdadera anticipación.

Así se expresará en la tercera estrofa, donde la escena se clarifica en lo que parece ser la intervención médica a un cuerpo en la obscuridad de la noche, "sous la fragile clarté d'une lampe", junto a "trois femmes et un homme" "cherchant à recoudre des corps". Se insinúa una escena en la memoria del sujeto lírico que recuerda un acontecimiento bélico, contextualizado en "ces nuits lointaines (qu'une fois de plus embrasait la guerre)". La escena, entonces, parece indicar las condiciones de visibilidad en las que se desarrolla dicha intervención médica sobre los cuerpos abatidos en combate, "que d'autres au dehors sans relâche déchiraient"; el "tacto" físico de la noche y el de los cuerpos que son tratados apuntan una misma simulación, gestual. El poema sintetiza así el "simulacro" corporal de dos gestos "toucher la nuit" y "recoudre des corps". Pero es posible sostener que el efecto emocional da sentido al poema porque en la última estrofa se consolida la atmósfera de perplejidad anteriormente insinuada. A partir de la noche visual y "táctilmente" inasible, los cuerpos heridos y el ambiente bélico que subyace a la escena, se sugiere una extrañeza que connota inquietud y estremecimiento; esta es la "textura semántica" del poema que delinea toda su prosodia emocional.

De modo que decimos que el significado se halla aquí encarnado puesto que surge de la corporeización en los gestos físicos del sujeto lírico que explora la noche y a los pacientes que son intervenidos. Ello se expresa igualmente en diversos rasgos de toda la serie "Nuits", obra en la cual se verá al cuerpo interactuar con el entorno, desde el inicio hasta el final. Es en esa interacción donde ha de rastrearse el significado encarnado, pues como señala Johnson, "in order to have human meaning, you need 
a human brain, operating in a living human body, continually interacting with a human environment that is at once physical, social, and cultural. Take away any one of these three dimensions, and you lose the possibility of meaning: no brain, no meaning; no body, no meaning; no environment, no meaning" (2007: 155). Desde esta misma idea, en el siguiente ejemplo la emergencia del significado puede ser entendida a partir de los órdenes simbólicos de esas tres dimensiones del lenguaje:

\section{La brume de l'hiver enveloppe \\ le monde visible on ne voit \\ ni griffes ni rouge rosée \\ sur la peau écorchée des corps}

à peine un renflement des gris

duveteux rappelle la fureur

des dents et des nerfs au combat

et la très vieille douleur

où l'esprit creuse sans relâche

à la rencontre d'une eau vive -

mer retournée par les vents de l'aube

de fonds entrouverts sans mot sans amour

tant de douleur fouillée sous les blancs

de peaux et de pensées qu'abandonnent

les nuits de tourmente sur les rochers -

(Gaspar, 2001: 142) .

\footnotetext{
4"La bruma del invierno envuelve / el mundo visible no se ve / ni garras ni rojo carmesí / sobre la piel arañada de los cuerpos // apenas una protuberancia de grises / mullidos recuerda el furor / de los dientes y los nervios en combate / y el antiguo tan dolor / donde la mente cava sin descanso / buscando un agua viva - // mar devuelto por los vientos del alba / fondos entreabiertos sin palabra sin amor / tanto dolor cavado bajo los blancos / de pieles y pensamientos que abandonan / las noches de tormenta encima de las rocas -" $(P A, 142)$.
} 
Se hace necesario examinar cómo ocurre la traducción de estas dimensiones que señala Johnson a los niveles simbólicos que tejen la prosodia emocional del poema. Así, el entorno paisajístico se da aquí como una "brume de l'hiver [qui] enveloppe / le monde visible", trastornando la nitidez de la percepción visual del observador lírico. En un primer momento, "on ne voit ni griffes ni rouge rosée"; interesa la materialización de lo "no visto" concretado específicamente en "griffes" y "rouge rosée", pues indica que la percepción no se produce desde lo visual sino desde lo táctil, "sur la peau écorchée des corps"; "écorchée", entonces, por la "brume" que baña y raspa "la peau"; se muestra así que el invierno deja una impronta física en los “corps". La distorsión visual se manifiesta en ese entorno nebuloso e ininteligible en el que se dibuja "à peine un renflement des gris duveteux". No obstante, detrás de ese "monde visible" que "l’hiver enveloppe" se halla un "corps sentant" que percibe el paisaje desde lo que podría llamarse una suerte de encarnación emocional. Entorno, cuerpo y cerebro perciben y sienten el significado pues estamos ante un paisaje que se activa emocionándolo, justamente en un "renflement [qui] rappelle la fureur", pero decimos cuerpo emocionado porque se trata concretamente de "la fureur des dents et des nerfs au combat".

Ello permite afirmar que la prosodia emocional del poema no consiste en la enunciación de la emotividad como generalidad del sentir, es decir, de la emoción como estado abstracto de la disposición sensible del sujeto ante el mundo. Se trata de una prosodia emocional porque el poema pone en práctica un hipónimo afectivo; dice "fureur" y especifica "des dents et des nerfs". La emoción sería un hiperónimo del cual el poema ejemplifica una manifestación concreta. La emoción no es una general, sino particular. Se ilustra desde la especificidad de una "très vieille douleur" y así se da un sentido hipónimo a lo afectivo ("la douleur"), que no es cualquiera sino una "très vieille". Por otra parte, "la douleur", es ya en sí mismo un hipónimo del hiperónimo emoción.

Así, el poema brinda la posibilidad de que el sujeto lírico otorgue 
un valor determinado a una experiencia afectiva que es "très vieille" porque pertenece al pasado vivido del yo lírico, y por lo tanto es un valor insustituible por otro cualquiera: es una "douleur où l'esprit creuse" en la memoria emocional. Decimos insustituible o "inintercambiable" por algún otro, ya que el poema enuncia una emoción que se ancla en una experiencia de sentido particular, definida y propia de una situación que instala el "locus emocional". Es justamente esa cualidad hipónima de lo emotivo desde la que el poema configura su significado, y es precisamente esto lo que llamamos la prosodia emocional del poema, puesto que rige la “acentuación dialectal" del sentir lírico, su entonación afectiva, el "ritmo anímico" de la enunciación.

Esta cualidad hipónima de lo emotivo es además insustituible por cuanto tiene su fundamento en un vivir concreto: se trata de una emoción que no puede ser otra, pues obedece a una lógica sensible determinada que se arraiga en la experiencia vivida. En su decir lo emocional, el poema encarna una especificidad que correlaciona lo escrito-imaginado con lo real-sentido. De modo que es posible afirmar que emerge un significado porque se cumple la condición de que la emoción involucre a lo mental, como una "douleur où l'esprit creuse sans relâche à la rencontre d'une eau vive". Aquí, la "douleur" propicia una "rencontre", que ha de ser leída como encuentro y como conocimiento simultáneamente: es "rencontre" de "l'esprit" que encarnadamente re-conoce "une eau vive". Se trata, pues, de una semantización bottom-up de la experiencia.

Más aún, es posible leer ese "esprit [qui] creuse" como una entidad dinámica, si se tiene en cuenta que ejerce aquí un gesto físico, ("creuse[r]"); de manera que "esprit" constituye una proyección de experiencia encarnada y adquiere además valor de una cualidad en cierto modo sensomotora. Por ello, el pensamiento se encuentra en una "circunstancia emocional" fluctuante, que responde al entorno que impregna al lenguaje que nombra el paisaje.

Así lo muestra, en la primera mitad de la última estrofa, esa "mer retournée par les vents de l'aube / de fonds entrouverts sans mot sans 
amour": los versos describen la manera en la que el mar vuelve a las profundidades durante la mañana, al producirse el descenso de la marea; aparecen sorprendentemente sus "fonds entrouverts", pues el mar se ha replegado y ha dejado al descubierto la arena que cubría. De modo que "mer", "vents", "aube" y "fonds" se hallan regidos por la ausencia del lenguaje y de la emoción —-"sans mot sans amour", dice-. La ausencia de una coma enumerativa entre "mot" y "sans" acentúa la unión de esa yuxtaposición que conforman lenguaje ("mot") y sentimiento ("amour"). $\mathrm{Y}$ es que esos "fonds entrouverts" no son únicamente los de la "mer retournée", sino también los del sujeto mismo, aquellos que "l'esprit creuse à la rencontre d'une eau vive". De ahí que el relieve afectivo resulte preponderante en el trazo compositivo del entorno.

La prosodia emocional del poema-paisaje continúa en la segunda mitad de la última estrofa, donde vuelve a expresarse el embodiment: "tant de douleur fouillée sous les blancs / de peaux et de pensées qu'abandonnent / les nuits de tourmente sur les rochers". Decimos embodiment porque, por una parte, aparece una vez más la emoción (“douleur”) encarnada gestualmente ("fouillée"); y es que si la emoción está aquí corporeizada en tanto superficie susceptible de ser "cavada" es porque se encuentra representada como territorio de impronta, como memoria del cuerpo "sous les blancs". Pero además porque, por otra parte, el cuerpo ("peaux") y la mente ("pensées") se desplazan fisicamente — "abandonnent" dice- y en ese sentido son también dinámicos. Una posibilidad de lectura se abre aquí: las "nuits de tourmente" "abandonnent" a "peaux" y "pensées". Más allá del evidente dinamismo que denota esta posibilidad, se da la circunstancia de que pone de relieve el hecho de que la palabra "tourmente" posee valor de perturbación atmosférica, $-\mathrm{y}$ no el de adversidad emocional, "tourment"- Sucede entonces que en el entorno espacial del poema paisaje ("nuits"), la emoción "se vierte" en forma de "tourmente" ambiental sobre la materia del espacio ("sur les rochers"). Por ello es posible afirmar que las "nuits de tourmente" activan un paisaje "emocionalmente encarnado"; ello sin obviar que "Nuits" es justamente el nombre de la serie de poemas 
que nos ocupa.

Cabe insistir en la cuestión del embodiment del poema atendiendo a diversas perspectivas, ya que el mismo Johnson fundamenta la idea del significado encarnado desde las bases neuronales de la conceptualización, según señala:

From a neural perspective, we need to know what concepts are and how they work. The obvious challenge for a theory of embodied meaning is twofold. First, if concepts are not disembodied symbolic representations, then what are they, from a neural perspective? Second, can all concepts be embodied, that is, grounded in sensorimotor aspects of experience? This second question requires an account of both concrete concepts (i.e., concepts of concrete objects, persons, events, and bodily actions) and abstract concepts (e.g., mind, knowledge, ideas, justice, rights, freedom) that are not about concrete, physical entities. My argument progresses in two steps. First, we must examine evidence that the meaning of concrete concepts is grounded in activations of sensorimotor areas of the brain. Second, we must suggest how abstract concepts (concepts not tied to specific sensory experiences) also rely on sensorimotor areas of the brain and are thus embodied (Johnson, 2007: 157).

En efecto, la distinción que plantea Johnson entre conceptos concretos y conceptos abstractos puede tener rentabilidad teórica en el análisis literario. Así, en el poema que nos ocupa sería posible contabilizar los siguientes conceptos concretos: "brume", "hiver", "enveloppe", "monde", "griffes", "rouge" "peau", “corps", "renflement”, “dents", "nerfs", “combat", “creuser", "rencontre", "eau”, "mer", "vents", "fouiller" "abandonner" y "nuits". Asimismo, los siguientes conceptos abstractos: "fureur", “douleur", "pensées", “esprit”, “amour”. Si bien no es del interés central de este estudio, estos últimos casos requerirían un análisis pormenorizado singular, ya que según la connotación y tratamiento 
que posean podrían denominar — o tener raíz en- conceptos concretos. Tal es la sugerencia de Johnson que aquí suscribimos y que refrendamos a través de nuestro análisis: que el pensamiento dicho "abstracto" posee relación última con la percepción, el movimiento y el sentimiento del cuerpo humano. De ahí que esta sea la base elemental del significado, y por ello sea posible brindar una dimensión epistemológica - conceptualabstracta - en la gestualidad, tal como se expresa en la prosodia emocional de los poemas de "Nuits", donde la encarnación rige el sentido último del texto.

Asimismo, Mark Johnson disiente de la idea de que los conceptos sean representaciones mentales aisladas, inscritas en el pensamiento del sujeto a partir de las "cosas del mundo". Uno de los argumentos principales de la perspectiva no-representacional de Johnson es que:

[...] concepts are not inner mental entities thatre-present external realities. Rather, concepts are neural activation patterns that can either be "turned on" by some actual perceptual or motoric event in our bodies, or else activated when we merely think about something, without actually perceiving it or performing a specific action. So in order to understand what concepts are from a neural perspective and how they work, we must examine how they emerge in the process of an embodied organism's ongoing engagement with its environment (Johnson, 2007: 157).

A la luz de esta consideración, retomaremos la reflexión y ejemplificación que propone Mark Johnson, a través de la observación de otro poema de "Nuits".

là où se creuse la vague

retient son souffle la crête

ce qui tente à nouveau de naître

tenu fermement dans la griffe 
de la peur sur elle-même fermée

et le frisson court de nervure

en nervure de soleil en ténèbres

poignées de couleurs dansées

jamais vues, que peint une musique

inconnue -

et toute l'encre de la vieille Chine

diluée, tremblée, bue par la brume

seul le trait continue de voler -

(Gaspar, 2001: 143) .

En el inicio del poema destaca, junto al crecimiento de "la vague", el verbo "retient" que se sujeta a "la crête", dando la imagen de una suerte de "suspensión" en el punto alto de la ola, que se halla de algún modo "contenida" en "son souffle ret[enu]". Interesa fundamentalmente que se metaforice esa retención de la ola suspendida a partir de la analogía de la respiración contenida — de hecho, este será el campo semántico en el que incidirá, dos versos más tarde, el verbo "tenu fermement"- De manera que el movimiento mediante el cual "retient son souffle la crête" reposa sobre la imagen de un gesto corporal —el de la respiración — que guarda tanto la contención como el potencial de acción, es decir, "ce qui tente à

\footnotetext{
5 "allá donde se ensancha la ola / retiene su suspiro la cresta / lo que intenta de nuevo nacer / sostenido firmemente a la garra / del temor en sí mismo encerrado / y el escalofrío corre de nervadura / en nervadura el sol en tinieblas / puñados de colores agitados / nunca vistos, que pintó una música / desconocida - // y toda la tinta de la antigua China / diluida, temblorosa, bebida por la bruma / sólo el trazo sigue volando — " (PA, 143).

${ }^{6} \mathrm{El}$ diccionario Larousse proporciona los siguientes valores a la acepción sustantivada de la palabra: "(1) Onde forcée produite par effet de friction du vent à la surface de la mer ou d'un lac. (2) Mouvement d'ondulation de la chevelure (surtout pluriel). (3) Masse d'air chaud ou froid qui arrive brusquement. (4) Phénomène d'ampleur assez considérable qui apparaît subitement. (5) Masse importante de personnes qui se déplacent ensemble. (6) Manifestation ample et subite d'un sentiment" (Larousse). Retendremos, a efectos de nuestro análisis, las acepciones 1 y 6 .
} 
nouveau de naître".

Se observa que en aquello que se halla "tenu fermement" subyace la posibilidad del movimiento - movimiento, por otra parte, tan inherente a la "vague" - . Esta entidad dinámico-retenida se encuentra "tenu fermement dans la griffe / de la peur sur elle-même fermée"; dichos dos versos resultan de interés en nuestra lectura ya que puntualizan la dimensión emocional que está detrás de ese "dinamismo-contenido"; se sugiere que el movimiento es retenido por un temor que paraliza; es decir, que la emoción es capaz de bloquear o impedir un instante la moción, capaz de "retener la cresta de la ola en su suspiro". Y es que, además, se da la circunstancia de que el concepto de "griffe de la peur" es él mismo paralizante, por cuanto la garra de un animal es una extremidad que gestualmente inmoviliza, por ejemplo, en el ataque; se diría entonces que el temor se metaforiza como extremidad anatómica ("griffe") que suspende el movimiento físico "sur elle-même fermée" — encarnada, entonces - En este sentido, "la peur sur elle-même fermée" sugiere que la emoción es capaz de encerrar sobre sí misma, de inmovilizar — “fermer" incrementa aún más el campo semántico de "retient", "tenu", etc.-.

Sin embargo, en seguida se producirá un giro en esta semántica de la inmovilidad que mostrará hasta qué punto el poema gaspariano conoce los procesos del sistema emocional. Y es que el temor aparece en el texto como entidad "dinámico-retenida", cuyo "frisson court de nervure en nervure". Así, "la peur" no constituye únicamente una paralización "sur elle-même fermée" sino también un estremecimiento que recorre el cuerpo "de nervure en nervure". Resulta destacable la idea de que el "frisson" ejerce una suerte de mecanismo de contracción, ya que tiene tanto potencial de paralización (“tenu fermement”) como de movilización ("nervure en nervure"); hace reaccionar al desplazarse a lo largo del cuerpo conteniéndolo, pero también agitándolo "de soleil en ténèbres".

En este punto del poema se produce un interesante giro en la somatización emocional del paisaje, ya que se acentúa también un valor visual de ese "frisson". Junto a lo gestual cobrará fuerza lo perceptivo, en 
esos "poignées de couleurs dansées" que sin embargo se formalizan en el espacio visual del observador lírico como "jamais vues". Surge así un contrasentido perceptivo que produce una tensión entre lo visto ("poignées de couleurs") y lo no visto ("jamais vues").

Parecería que lo emocional llega a la percepción visual; sin embargo, se revela que el estímulo detonante del miedo es en realidad auditivo. Así lo muestra el verso: "que peint une musique / inconnue", donde el sonido del mar — su "musique" - ha pintado todo un paisaje emocional. "Pintado", porque el temor parece ser trazado por esa "musique / inconnue". La intermodalidad — táctil, visual, sonora - con que se da el tratamiento del miedo en el lenguaje poético sugiere que la experiencia emocional está siendo resultado de otra experiencia no explícita en el poema, donde repentinamente aparece una contextualización geográfica: "toute l'encre de la vieille Chine / diluée, tremblée, bue par la brume". Se diría que los contrasentidos y contracciones antes señalados provienen del hecho de que este poema-paisaje representa una experiencia de contemplación, acaso más propiamente de un elemento pictórico que de un paisaje directamente "vivido". Ilustrado, en efecto, con un trazo que se muestra "diluée", "tremblée" y "bue"; el paisaje se ve dibujado como la emoción misma del temor que describe el poema, y que por ello subyace a ambas representaciones verbal y pictórica. De manera que en "seul le trait continue de voler", ese "trait" es auténticamente el del pintor "de la vieille Chine" que sugiere el paisaje donde "se creuse la vague", "retient son souffle la crête" y "seul le trait continue de voler".

Así, entonces, si bien no se descarta que el poema pueda ejemplificar un ejercicio de écfrasis sobre una pintura o un dibujo, sí conviene considerar que, con independencia de ello, se retrata aquí una experiencia subjetiva de contemplación del entorno que es "activada" por la lectura emocional de los estímulos paisajísticos que ahí se representan. En este contexto, conviene una puntualización de Joseph LeDoux acerca del "inconsciente cognitivo": 
[...] il vaut mieux considérer les états émotionnels subjectifs, à l'instar des autres états de la conscience, comme le résultat d'un traitement inconscient de l'information. De même que nous pouvons étudier comment le cerveau traite inconsciemment l'information dans la perception des stimuli visuels et l'utilise pour orienter le comportement, nous pouvons aussi étudier comment le cerveau agit pour donner une signification émotionnelle aux stimuli. Et de même qu'en étudiant comment le cerveau traite les stimuli visuels, nous espérons mieux comprendre comment il crée l'expérience de perception subjective qui l'accompagne, nous espérons aussi que l'étude de la manière dont le cerveau traite l'information émotionnelle nous aidera à mieux comprendre comment cela peut être à l'origine des expériences émotionnelles (Ledoux, 2005: 40).

Desde esta misma lógica, la cognición encarnada dialoga con las investigaciones del neurofisiólogo Vittorio Gallese - conocido por sus estudios sobre las neuronas espejo- acerca del "interactional process", que describe el modo en que los intercambios de energía entre un organismo y su entorno poseen un correlato en patrones de activación neuronal (Johnson, 2007: 157). Recuperamos, pues, la síntesis de Vitorio Gallese:

If we analyse at the physical level of description the relationship between biological agents and "the world outside", we will find living organisms processing the different epiphanies of energy they are exposed to: electromagnetic, mechanical, chemical energy. Energy interacts with living organisms. It is only by virtue of this interaction that energy can be specified in terms of the "stimuli" (visual, auditory, somatosensory, etc.) to which every organism is exposed. The result of the interaction between energy, now "stimulus", is translated, or better, transduced into a common informational code. The receptors of the different 
sensory modalities are the agents of the transduction process: they convert the different types of energies resulting from organismsworld interactions into the common code of action potentials. Action potentials express the electro-chemical excitability of cells, and constitute the code used by the billions of neurons that comprise the central nervous system to "communicate" with each other (Gallese, 2003: 1232, apud Johnson, 2007: 158).

Del interactional process, a la teoría del significado encarnado de Johnson le interesa el hecho de que las interacciones entre el organismo y el entorno transforman patrones de energía del espacio en estímulos para el organismo: tal como señala, "these patterns are converted within the organism to action potentials in neurons, thus initiating vast neuronal "communication"” (Johnson, 2007: 158). Sostiene Johnson que la razón por la que Gallese denomina a esos patrones representations, es que estos codifican en cierto modo potenciales de acción neuronal, pero ello no significa que se trate de representaciones de tipo proposicional que relacionan entidades mentales internas con realidades externas directas. Al contrario, "Gallese suggests that these activation patterns should be thought of as both the vehicle of representation and the content, because, as patterns of organism-environment interaction, they are both the structures of the interactions themselves (the vehicle) and simultaneously what we might call "models" of the world for the organism (the content)" (Johnson, 2007: 158). Para el filósofo cognitivo será más importante el peso de la interacción que el de la representación.

Desde esta consideración, obsérvese ahora un último poema de "Nuits":

"Ô Mère, ô nuit ma mère qui m'enfantas"

d'une infinie patience il te faut réapprendre

à aimer un feu comme naguère perdu au loin 
t'arrêtant à la tombée du jour

quelque part dans l'inconnu d'un désert -

tu demandes rien que pouvoir toucher

par instants la chaleur d'un battement

léger sillage d'un oiseau

qui rompt le cercle du regard

tes yeux verront-ils la clarté encore?

elle sourit au présent tout autour

tel l'Orient en toi du vol nocturne

des grands et des infimes migrateurs -

le jour revenu accueille les fruits

des forages nocturnes qui éclatent

dans la pièce nocturne de Schumann

qu'il demande de jouer simplement -

dans le pinceau de T'ang parti sans bruit

en septembre, encre instantanée

du clair jaillissement de vivre,

dans le noir presque bleu des nuits d'automne-

voici encore ces cathédrales d'ailes

dans l'air du soir criblé de cris

légèreté de ce jeu ô combien précis

dans la trame sans bornes du mouvoir,

désir de dessiner l'air transparent

soudé au-dedans à un rien de braise -

peu à peu en elle-même sombre la mer 
dans l'unique foulée d'un trait de pinceau

et j'écoute longuement dans le noir

le bruit de l'eau, ma seule pensée -

(Gaspar, 2001: 152-153) ${ }^{7}$.

El contenido que rige la prosodia emocional del poema se manifiesta en la declaración de un sujeto lírico que se dirige a otro: "il te faut réapprendre / à aimer un feu comme naguère perdu au loin". Se evoca una suerte de memoria emocional ("naguère") de la segunda voz lírica pues trae un punto anterior - "perdu au loin"- de la experiencia amorosa. Decimos que "aimer" posee aquí una cualidad perceptiva porque "un feu [...] perdu au loin" hace referencia a la contemplación acaso de la luz "quelque part dans l'inconnu d'un désert": esa contemplación tiene sobre la segunda voz lírica un efecto paralizante —emoción entonces"t'arrêtant à la tombée du jour", dice. Razón por la cual se simboliza tanto un "feu" visual como un "feu" emocional; de ahí que "réapprendre à aimer" pueda ser leído como un "réapprendre" a contemplar y a sentir, es decir, como interacción del organismo con el entorno, cuyas "improntas" quedan en la memoria del sujeto lírico.

A continuación, la emoción cobrará aspecto táctil cuando la voz

\footnotetext{
7“' Oh Madre, oh noche madre que me engendras' // con una paciencia infinita tienes que reaprender / a amar un fuego como no hace tanto perdido a lo lejos / deteniéndote al atardecer / en alguna parte de lo desconocido de un desierto - // no pides más que poder tocar / por instantes el calor de un golpeteo / ligero trazo de un ave / que rompe el círculo de la mirada // ¿verán tus ojos la claridad de nuevo? / ella sonríe ahora alrededor / como en ti el Oriente del vuelo nocturno / de las grandes y diminutas aves migratorias - // el día devuelto acoge las frutas / perforaciones nocturnas que deslumbran / la habitación nocturna de Schumann / que pide sencillamente tocar - // en el pincel de T'ang se fue sin ruido / en septiembre, tinta instantánea // de la clara emanación de lo vivo, / en el negro casi azul de las noches de otoño - // de nuevo aquí estas catedrales de alas / en el aire de la tarde acribillada a gritos / ligereza de ese día oh cuán exacto / en la trama sin contornos del movimiento, / deseo de dibujar el aire transparente / soldado por dentro a una nada que calcina - // poco a poco en sí mismo sombrío el mar / en la única zancada de un trazo de pincel / y escucho largamente en el negro / el ruido del agua, mi único pensamiento-" $(P A, 152-153)$.
} 
lírica advierta: "tu demandes rien que pouvoir toucher / par instants la chaleur d'un battement / léger sillage d'un oiseau / qui rompt le cercle du regard". La relación de "toucher", "chaleur" y "regard" acentúa un ejercicio de contemplación que es guiado por el placer estético, pero cuyo valor efímero es metaforizado en la imagen del breve paso de un ave en el campo visual del yo lírico. Es "la chaleur d'un battement" cuyo contacto imposible se da apenas a través de la mirada. De ahí que la pregunta del observador lírico resulte relevante en la interacción organismo-entorno: "tes yeux verront-ils la clarté encore ? / elle sourit au présent tout autour / tel l'Orient en toi du vol nocturne / des grands et des infimes migrateurs". En efecto, la transición de día-noche-día es el espacio ambiental del poema ("la tombée du jour", "du vol nocturne", "le jour revenu", "forages nocturnes") donde se da el viaje "des grands et des infimes migrateurs" que el sujeto contempla "dans la pièce nocturne".

Asimismo, en el desenlace del texto, lo obscuro deviene perceptible: “j'écoute longuement dans le noir / le bruit de l'eau, ma seule pensée". Estas son las "energías del espacio" que fundan la experiencia de sentido del sujeto. Efímeras, como se muestran la emoción y la percepción, el poema-paisaje es entonces - a semejanza del lienzo - auténtica "encre instantanée”. Y ello constituye una conclusión poetológica que es plausible teniendo en cuenta los principios de Johnson, ya que para la teoría de la cognición encarnada el fundamento del sentido reside en esa interacción dinámica espacio-organismo, en la cual se producen los patrones de los conceptos encarnados. Así:

Our neural makeup, in interaction with the energies available in our surroundings, will result in specific neural connections and tendencies for specific neuronal clusters to fire in the presence of certain specific stimuli. Concepts have to be understood as the various possible patterns of activation by which we can mark significant characteristics of our experience (Johnson, 2007: 160). 
Por lo tanto, en un nivel cerebral, el sentido emergería de las relaciones de estos patrones de energía que favorece la dinámica de las interacciones sujeto-espacio. Si bien aún no se cuenta con suficiente investigación acerca de los patrones neuronales de los conceptos que así se producen, algunas de las propuestas más interesantes son las de Gallesse y Lakoff, que han sugerido que:

[...] conceptualization uses some of the very same sensorimotor processes that are activated in actual perceptual and motor experiences. They thus reject any view that regards thinking as a kind of cognition radically different from perceiving and doing. What I find most exciting about their theory is that it suggests some ways in which even abstract concepts are tied to sensorimotor processes. Gallese and Lakoff discuss experiments with both monkeys and humans showing that sensorimotor areas involved in perception and action are also activated in conceptualizing those perceptions or actions, and they then try to extend this account to abstract concepts (Vittorio Gallesse y George Lakoff en Johnson, 2007: 160).

Cabe concluir, entonces, que los procesos de conceptualización y de abstracción —entendida esta como la capacidad de categorizar y caracterizar la experiencia en unidades de representación mentalconstituyen objetos potenciales de estudio cognitivo-literario. Pero un objeto de estudio singular que invita a la elaboración de análisis que aúnen perspectivas neurobiológicas y filosóficas con el propósito de brindar a la teoría literaria instrumentos novedosos de interpretación poética. Por ello, resulta plausible proponer indagaciones interdisciplinarmente informadas hacia el pensamiento poético que examinen, desde la exégesis del texto, las estructuras y operaciones del pensamiento literario. Labor sobre la cual se espera que la teoría de la literatura aporte ideas fructíferas. 


\section{REFERENCIAS BIBLIOGRÁFICAS}

ARMSTRONG, P. B. (1990). Conflicting Readings: Variety and Validity in Interpretation. Chapel Hill: University of North Carolina Press. (2013). How Literature Plays with the Brain. The Neuroscience of Reading and Art. Baltimore: Johns Hopkins University Press.

BAYNE, T. \& MONTAGUE, M. (eds.) (2014 [2011]). Cognitive Phenomenology. Oxford: Oxford University Press.

BURKE, M. \& TROSCIANKO, E. (eds.). (2017). Cognitive Literary Science. Dialogues between Literature and Cognition. Oxford: Oxford University Press.

DEHAENE, S. (2007). Les neurones de la lecture. París: Odile Jacob.

FISH, S. (1980). "Literature in the Reader: Affective Stylistics". En Reader-Response Criticism, J. Tompkins (ed.), 70-100. Baltimore: Johns Hopkins University Press.

FREEMAN, M. (2008). "Reading Readers Reading a Poem: From Conceptual to Cognitive Integration". Cognitive Science 2, $102-$ 128.

GASPAR, L. (2004 [2001]). Patmos et autres poèmes. París: Gallimard.

GILlES, F. \& TURNER, M. (2003). The Way We Think: Conceptual Blending and The Mind's Hidden Complexities. New York: Basic Books.

GILLIOZ, C. (2013). The specificity of emotion inferences in text comprehension: the role of top-down and bottom-up processes. Thèse de doctorat. Fribourg: Université de Fribourg.

ISER, W. (1980). The Act of Reading: A Theory of Aesthetic Response. Baltimore: Johns Hopkins University Press.

JACOBS, A. (2015). "Neurocognitive poetics: methods and models for investigating the neuronal and cognitive-affective bases of literature reception". Frontiers In Human Neuroscience 9, 186.

JOHNSON, M. (2007). The Meaning of the Body: Aesthetics of Human Understanding. Chicago: University of Chicago Press. 
LEDOUX, J. (2005). Le cerveau des émotions. París: Odile Jacob.

TYSON, L. (2006). Critical Theory Today: A User-Friendly Guide. London: Routledge.

Recibido el 16 de agosto de 2018.

Aceptado el 6 de septiembre de 2018. 
